\title{
Acquired hemophilia as first manifestation of breast carcinoma in a man under long-term spironolactone therapy
}

\begin{abstract}
A 69-year-old man under long-term spironolactone therapy (16 years) was hospitalized with spontaneous hematoma on the trunk and extremities. Coagulation studies disclosed an acquired hemophilia that was successfully treated with human factor VIII for a few days and immunosuppressive agents for several months. Physical examination revealed bilateral gynecomastia and an upper left quadrant breast nodule. Complete staging was unremarkable. Complete left mastectomy was performed. Histopathology showed invasive ductal carcinoma, expressing positivity for estrogen and progesterone receptors. The acquired hemophilia was considered to be a paraneoplasic syndrome. The question of a linkage between long-term spironolactone therapy and breast carcinoma is discussed.
\end{abstract}

Key words Acquired hemophilia - Breast carcinoma . Spironolactone

\section{Introduction}

Acquired hemophilia is a rare disease, considered to be a paraneoplasic syndrome in nearly $10 \%$ of cases. ${ }^{1}$ Male breast carcinoma accounts for nearly $1 \%$ of all diagnosed breast carcinomas. ${ }^{2,3}$ Unfortunately, male breast cancer is

O. Lamy $(\bowtie) \cdot$ H. Elmiger

Service of Medicine A, Department of Internal Medicine, University Hospital, CHUV, BH10, CH-1011 Lausanne, Switzerland

Tel. +41.21.314.02.81; Fax +41.21.314.08.71

e-mail: olivier.lamy@chuv.hospvd.ch

M. Fiche

Department of Pathology, University Hospital, Lausanne, Switzerland

\section{J. Bauer}

Oncology Center, Department of Internal Medicine, University

Hospital, Lausanne, Switzerland

F. Livio

Division of Clinical Pharmacology, Department of Internal

Medicine, University Hospital, Lausanne, Switzerland often diagnosed at a late stage because of the minimal awareness of presenting symptoms by the patient and sometimes by the health-care provider. Because of this late presentation, the overall prognosis is less favorable., ${ }^{2,3}$ Spironolactone has antiandrogenic characteristics, and animal data suggest the oncogenic potential of spironolactone's metabolite. We report a very rare case of a man under long-term spironolactone therapy who was hospitalized for hematoma as the first manifestation of an acquired hemophilia related to breast carcinoma.

\section{Case report}

A 69-year-old man with a history of membranous nephropathy and moderate renal failure (creatinine clearance, $50 \mathrm{ml} / \mathrm{min}$ ) had been on spironolactone therapy $(100 \mathrm{mg} /$ day) for 16 years. He was known to have atrial fibrillation, hyperlipidemia, and diabetes. Co-medications included furosemide, digoxin, acenocoumarol, simvastatin, and glibenclamide. He was hospitalized on September 12, 2001 with arthralgia and spontaneous hematoma on the trunk and extremities.

At presentation, his hemoglobin level was $88 \mathrm{~g} / \mathrm{dl}$, white cell count was $17000 / \mu 1$, and platelet count was $36.4 \times 10^{4} /$ $\mu \mathrm{l}$. Complete chemistry confirmed an elevated creatinine level, at $120 \mu \mathrm{mol} / \mathrm{l}$ (normal range, 44-106 $\mu \mathrm{mol} / \mathrm{l}$ ) and revealed only a slight increase of total creatine kinase and lactate dehydrogenase. Urinanalysis was normal. Coagulation studies disclosed: prothrombin time, 130\% (normal range, $85 \%-125 \%$ ); activated partial thromboplastin time, $91 \mathrm{sec}$ (normal range, 21-33 sec); fibrinogen, $5.8 \mathrm{~g} / 1$ (normal range, 1.6-3.8g); negative fibrin monomer; D-dimer, 0.4$0.8 \mathrm{mg} / 1$ (normal range, $<0.2 \mathrm{mg}$ ). Factor VIII was very low, at $1 \%$ (normal range, $70 \%-150 \%$ ) and an inhibitor was identifiable.

An acquired hemophilia was diagnosed. Fresh frozen plasma was initiated upon presentation. Once factor VIII inhibitor was discovered, human factor VIII was started, at a dose of $200 \mathrm{UI} / \mathrm{kg}$ per day. This treatment was given at a 


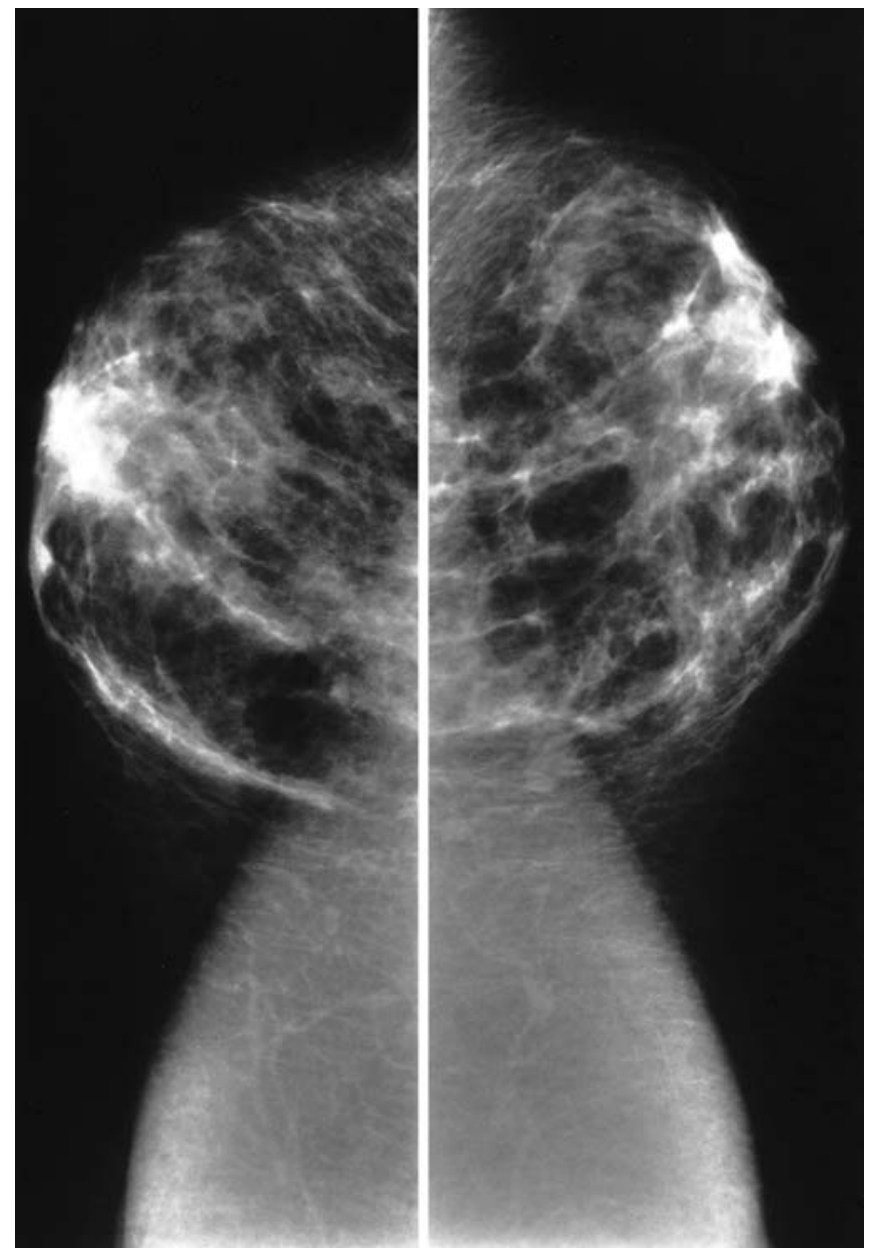

Fig. 1. Mammography. Mass with irregular contours (diameter, $1.1 \mathrm{~cm}$ ) without calcifications, at the junction of the upper and the lower outer left quadrant of the left breast

tapered dose, according to the clinical status and the normalization of factor VIII values, for a period of 16 days. Immunosuppressive agents, including cyclophosphamide $200 \mathrm{mg} /$ day and prednisone $1 \mathrm{mg} / \mathrm{kg}$ per day $(80 \mathrm{mg})$ were started with human factor VIII. Considering the good biological response, prednisone was decreased to $0.5 \mathrm{mg} / \mathrm{kg}$ per day after 2 weeks. Results of serum protein electrophoresis; serologies for virus, including HIV, hepatitis virus, cytomegalovirus, and Epstein-Barr virus; and rheumatologic tests, including rheumatoid factor, antinuclear antibody, and antineutrophil cytoplasmic antibodies, were unremarkable. Complete examination revealed bilateral gynecomastia and an upper left quadrant breast nodule of $2 \times 3 \mathrm{~cm}$. The gynecomastia had appeared after the initiation of spironolactone, and had been known by the patient and his general practitioner for 10 years or more. No physical examination of the gynecomastia had been made previously. The mammographic findings are shown in Fig. 1.

An ultrasound-guided needle-core biopsy was performed on September 28. Histopathological analysis showed invasive ductal carcinoma. Staging, including thoracic, abdominal, and pelvic computed tomography $(\mathrm{CT})$

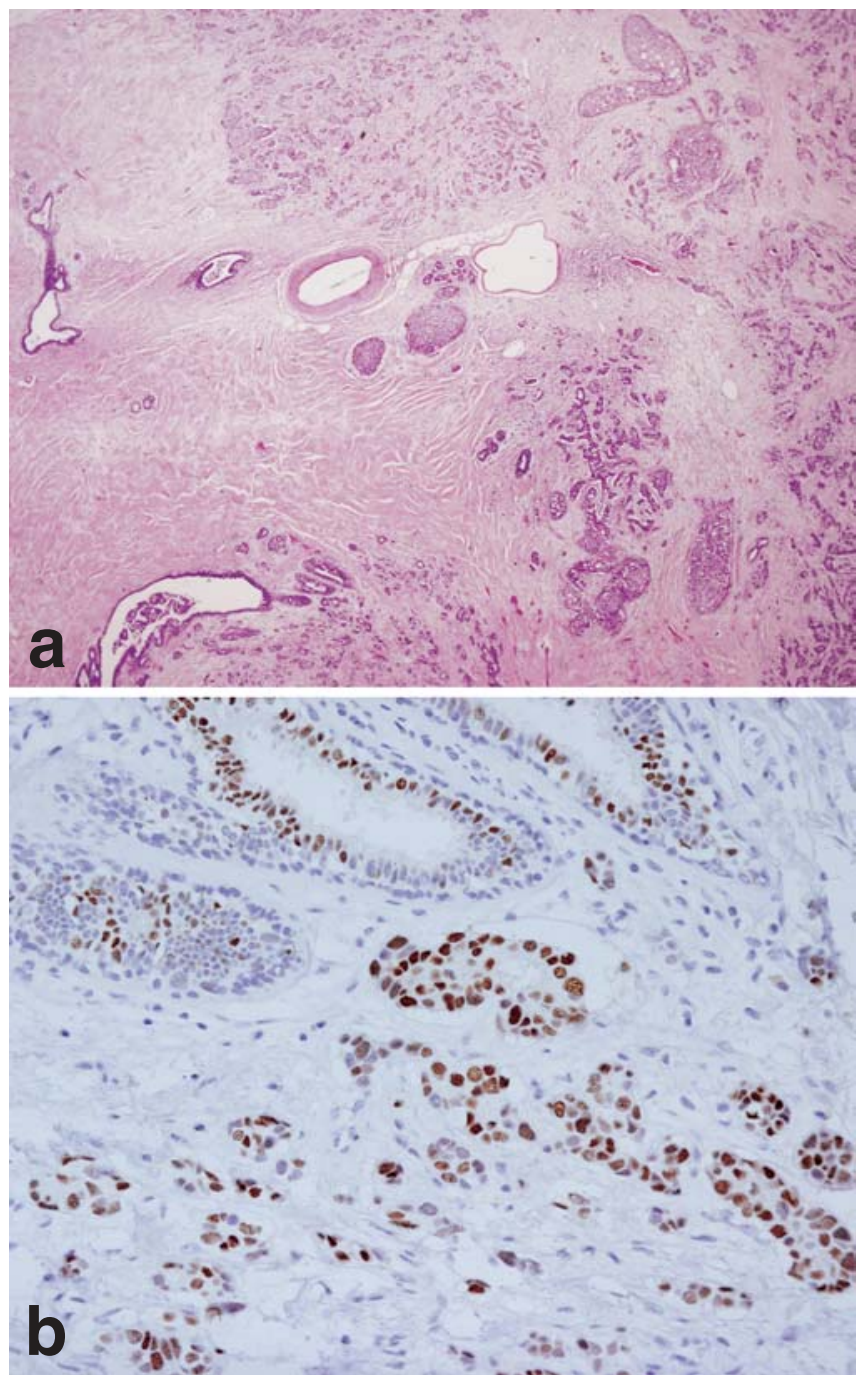

Fig. 2a,b. Invasive ductal carcinoma, histopathology. a Invasive ductal carcinoma with minimal in situ component (right), and fibrotic peritumoral mammary tissue consistent with the fibrous type of gynecomastia (left). b Immunohistochemical staining, showing that $100 \%$ of the invasive carcinoma cells, as well as some normal duct epithelial cells $(t o p)$, express estrogen receptor. a H\&E, $\times 200$; b immunoperoxidase, $\times 400$

scanning; bone scintigraphy; and laboratory parameters (corrected calcemia, liver function tests) was unremarkable. A mastectomy was performed on October 15, without complication. At gross examination, the tumor measured $1.8 \mathrm{~cm}$. Histologically, it was invasive ductal carcinoma with an in situ ductal carcinoma component (Fig. 2a,b). The invasive carcinoma was organized partly in tubes $(50 \%$ of tumor surface), nuclear pleomorphism was marked, and the mitotic count was low (six mitoses per ten high-power fields), corresponding to histoprognostic grade II according to the Elston and Ellis system. The in situ component accounted for $10 \%$ of the tumor surface, was located within the tumoral nodule, and exhibited low nuclear grade and a cribriform pattern. All (100\%) tumor cells, in both components, expressed estrogen receptors (Fig. 2b) and progesterone receptors. Peritumoral mammary tissue exhibited diffuse 
fibrosis surrounding ducts, without significant epithelial hyperplasia, consistent with the fibrous type of gynecomastia (Fig. 2a). Two axillary sentinel lymph nodes were analyzed on 12 sections $250 \mu \mathrm{m}$-thick, after both hematoxylin eosin and immunohistochemical stainings, the latter using an antibody directed to cytokeratins. No tumor cells were found by these procedures. The final pathological diagnosis was invasive ductal carcinoma, grade II, pathological stage pT1pN0 (sn). Spironolactone was stopped and adjuvant tamoxifen started. The patient returned home on October 23. Blood test results rapidly returned to normal and the immunosuppressive therapy was stopped after 7 months. The factor VIII level was in the upper normal range and factor VIII inhibitor was undetectable. After 2 years, the patient remains clinically well, without clinical or biological recurrence. At physical examination, the size of the right gynecomastia has decreased and the consistency is more adipose.

\section{Discussion}

Acquired hemophilia is a rare disease caused by the development of auto-antibodies against factor VIII. In approximately $50 \%$ of cases, the etiology is unknown. ${ }^{1}$ Autoimmune disorders account for more than $10 \%$ of cases, paraneoplasic syndrome for $7 \%-15 \%$ of cases, the third trimester of pregnancy or the postpartum period for $10 \%$ of cases, adverse drug reaction for $3 \%-6 \%$ of cases, and various skin disorders for $2 \%-5 \%$ of cases. ${ }^{1}$ The treatment of choice for acquired hemophilia includes the use of human or porcine factor VIII in patients who are bleeding, and immunosuppressive agents (corticosteroids with or without cyclophosphamide or chlorambucil).,

We performed a Medline search of the literature and a review of appropriate publications to identify a link between acquired hemophilia and spironolactone or breast carcinoma. No case of association between spironolactone and acquired hemophilia was described. Adverse drug reactions with penicillin, ampicillin, phenytoin, nitrofurazone, chloramphenicol, sulfa drugs, or fluoroquinolones have been associated with factor VIII inhibitors. ${ }^{1}$ Some case reports have suggested a relationship between chronic renal failure and acquired hemophilia. ${ }^{6}$ This association was not described in the review by Bossi et al. ${ }^{1}$ In our patient, the blood test results rapidly returned to norm after the immunosuppressive therapy was started and the left mastectomy performed. At the same time, the magnitude of chronic renal failure did not change. For this reason, we have not suggested a relationship between chronic renal failure and acquired hemophilia. We identified only three other cases describing an association between breast cancer and acquired hemophilia. ${ }^{7-9}$ These three cases occurred in women. In our patient, breast carcinoma was considered to be the "primum movens". A retrospective study of 41 patients with cancer and acquired hemophilia revealed that in $61 \%$ of cases solid-organ tumor was the underlying diagnosis. ${ }^{7}$ Prostate cancer was the most common, followed by lung carcinoma. The most common histologic type was adenocarcinoma.

Putative risk factors for breast carcinoma in men included advanced age, Jewish ancestry, a positive family history, excess exposure to female hormones (Klinefelter's syndrome), environmental exposure (irradiation), alcohol, and obesity. ${ }^{2,3}$ Our patient had none of these risk factors. Considering the well-known causal relationship between spironolactone and gynecomastia, although gynecomastia is not recognized per se as a predisposing factor for breast carcinoma, the question of a linkage between long-term spironolactone therapy and breast carcinoma was raised in this case. The manifestations of spironolactone's antiandrogenic effects are various and partly dose- and time-dependent; in men, these involve gynecomastia, declining libido, and impotence. Competitive antagonism on androgenic tissue receptors by spironolactone is thought to be the main mechanism. Weak inhibition of androgen synthesis and increased conversion of androgens to estrogens were irregularly demonstrated and are probably minor..$^{10,11}$ Animal studies have shown an increased rate of neoplasia, including breast carcinoma, in rats on high-dose canrenoate, another aldosterone antagonist which shares a common metabolite (canrenone) with spironolactone. ${ }^{12}$ Some cases of breast carcinoma in women under spironolactone therapy have been reported, but this association may only be fortuitous. ${ }^{13}$ Male cases have also been reported to the WHO database, without a proven causal relationship.

Although there is no proven association between spironolactone and breast malignancy, a doubt cannot be ruled out, considering the antiandrogenic characteristics of spironolactone and animal data suggesting the oncogenic potential of spironolactone's metabolite. As spironolactone is now widely used, especially for cardiac failure, special attention should be paid to its safety profile.

\section{References}

1. Bossi P, Cabane J, Ninet J, et al. (1998) Acquired hemophilia due to factor VIII inhibitors in 34 patients. Am J Med 105:400-408

2. Goss PE, Reid C, Pintilie M, et al. (1999) Breast carcinoma: a review of 229 patients who presented to the Princess Margaret Hospital during 40 years: 1955-1996. Cancer 85:629-639

3. Giordano SH, Buzdar AU, Hortobagyi GN (2002) Breast cancer in men. Ann Intern Med 137:678-687

4. Kleinman MB (2002) Anti-inhibitor coagulant complex for the rescue therapy of acquired inhibitors to factor VIII: case report and review of the literature. Haemophilia 8:694-697

5. Boggio LN, Green D (2001) Acquired hemophilia. Rev Clin Exp Hematol 5:389-404

6. Narukawa N, Abe T, Shouno M, et al. (1999) A case of factor VIII inhibitor-positive acquired hemophilia treated by plasmapheresis. Ther Apher 3:323-325

7. Sallah S, Wan JY (2001) Inhibitors against factor VIII in patients with cancer. Cancer 91:1067-1074

8. Lopez R, Mateo J, Cruz J, et al. (1995) Presentation of an acquired factor VIII inhibitor with a muscular haematoma mimicking a deep venous thrombosis in a patient with breast cancer. Blood Coagul Fibrinolysis 6:680-682

9. Barlas AH (1986) Acquired factor VIII deficiency in an elderly woman on tamoxifen. J Am Geriatr Soc 34:318-320 
10. Corvol P, Michaud A, Menard J, et al. (1975) Antiandrogenic effect of spironolactones: mechanism of action. Endocrinology 97:52-58

11. Miyatake A, Noma K, Nakao K, et al. (1978) Increased serum oestrone and oestradiol following spironolactone administration in hypertensive men. Clin Endocrinol 9:523-533
12. Wagner BM (1987) Long-term toxicology studies of spironolactone in animals and comparison with potassium canrenoate. J Drug Dev 1S2:7-11

13. Loube SD, Quirk RA (1975) Breast cancer associated with administration of spironolactone. Lancet 28:1428-1429 